\title{
Prevalent Congenital Anomalies and Metabolic Disorders among Live-Born Neonates in Hospitals in General Santos City, Philippines (2009-2012)
}

\author{
Florence L. Zapico, ${ }^{1}$ Adrian T. Penaflorida, ${ }^{2}$ Catherine Hazel M. Aguilar ${ }^{3}$ and Eillen Gay I. Palarpalar ${ }^{4}$ \\ ${ }^{1}$ Science Department, College of Natural Sciences and Mathematics, Mindanao State University-General Santos City, Philippines \\ ${ }^{2}$ College of Medicine, Davao Medical School Foundation Inc., Davao City, Philippines \\ ${ }^{3}$ Graduate School, University of the Philippines at Los Banos, Laguna, Philippines \\ ${ }^{4}$ General Santos City District Hospital, General Santos City, Philippines
}

\begin{abstract}
Objective. Birth defects are among the leading causes of infant mortality and morbidity in the Philippines. While affected infants make up a sizeable portion of live births in General Santos City (GSC), no information is available about their actual numbers. This study aims to fill the knowledge gap about the prevalence and nature of congenital anomalies (CAs) and congenital metabolic disorders (CMDs) in the city from 2009 to 2012.

Methods. A retrospective study of in-patient records from six (6) medical facilities was done for CA/CMD cases from 2009-2012. Among the CMDs tested were congenital hypothyroidism $(\mathrm{CH})$, congenital adrenal hyperplasia (CAH), galactosemia (GAL), hyperphenyalaninemia (HPA), phenylketonuria (PKU) and glucose-6-phosphate dehydrogenase deficiency (G6PD def).

Results. Collected data revealed 109 cases of CAs with limb deformities, oro-facial clefting and neural tube disorders comprising majority of cases. There were 878 reported cases of CMOs with glucose-6-phosphate dehydrogenase deficiency (G6PO def) as the most prevalent at 829 cases. There was also a preponderance of CAs/CMDs in a government hospital for the indigent.
\end{abstract}

Conclusions. These results underscore the emergence of CAs and CMDs as a major health problem among newborns in GSC. Higher incidences of birth defects in one district hospital also reveal a tentative link between $C A / C M D$ incidence and socioeconomic status. It is of paramount importance therefore, to undertake expansion of the newborn screening program and to establish local birth registries so that a more comprehensive and realistic picture of $C A / C M D$ prevalence in the city will be obtained.

Key Words: congenitat anomaties, congenital metabolic disorders, ive-borm neonates

\footnotetext{
Corresponding author: Florence Lasalita-Zapico, MSc

Science Department

College of Natural Sciences and Mathematics

Mindanao State University-General Santos City, 9500 Philippines

Telephone: +63833806555

Fax No.: +6383 3806576

Email: florence.zapico@gmail.com
}

\section{Introduction}

Congenital anomalies (CAs), defined as structural and functional abnormalities present at birth, are a major cause of infant mortality and childhood morbidity. ${ }^{1}$ Reports from the Philippines indicate that birth defects account for $11.2 \%$ of infant morbidity. ${ }^{2}$ Furthermore, infants born with CAs have very special needs which hospitals and medical facilities are oftentimes ill-equipped to address. Congenital metabolic disorders (CMDs) on the other hand, are inborn errors of metabolism resulting from single gene disorders which when left unchecked invariably cause either mental retardation or death of the affected patients. Since newborns with CMD appear to be normal at birth, early detection is vital in preventing the progression of symptoms of the disorder. In the Philippines, the Newborn Screening Reference Center is directly responsible for maintaining the national testing database and case registries. As of the time the study was conducted (2012), the CMDs being tested in General Santos City hospitals were congenital hypothyroidism $(\mathrm{CH})$, congenital adrenal hyperplasia $(\mathrm{CAH})$, galactosemia (GAL), hyperphenyalaninemia (HPA), phenylketonuria (PKU) and glucose-6-phosphate dehydrogenase deficiency (G6PD def).

In 2008, the Philippine Birth Defects Surveillance Group was launched to establish a surveillance system for congenital anomalies and their associated risk factors. Starting with 32 participating facilities, it had expanded into 64 facilities in 2010. In GSC, a study was conducted by Zapico et al on congenital anomalies among live-born neonates from 2006 to 2008. ${ }^{3}$ The study, however, was exploratory in nature and focused more on inborn metabolic disorders and to a limited extent tackled congenital malformations and chromosomal abnormalities observed in the newborn.

The impact of CA/CMD on the pediatric population in particular and the paucity of information about its prevalence have made the study timely and relevant. This present research therefore seeks to address this problem regarding scanty data on $\mathrm{CA} / \mathrm{CMD}$ incidence and to fill the knowledge gap vis $a$ vis the prevalence trends of these congenital anomalies in GSC from 2009 to 2012. This is especially important since General Santos hospitals provide 
medical services to other areas in South Cotabato and Sarangani Province. Finally, studies such as this will provide information about CA/CMD incidence in this area of the Philippines where no such data is available and there is no facility or agency for their ascertainment.

\section{Methods}

General Santos, which is found at the southernmost tip of the Philippines, is a highly urbanized city with 53,606hectare land area and is considered as the $15^{\text {th }}$ most populous city in the country having a population of 538,086 as of 2010.4 General Santos City is part of the SOCCSKSARGEN (South Cotabato, Cotabato, Sultan Kudarat, Sarangani Province and General Santos) region and has 26 barangays of which half can be considered urban. The availability of jobs, opportunities for leisure, business and education, and the accessibility of the city via sea, land and air transport resulted in increasing migration and in the influx of daily transients. Using current population growth rate $(3.53 \%)$, it can be projected that the population will increase from 658,834 in 2011 to 864,388 in 2017. As a consequence of economic development, rapid population growth, uncontrolled urban sprawl and socio-economic problems are beginning to take hold of the place. Studies such as this therefore provide baseline data about CA/CMD incidences in a medium-sized city such as GSC which is on the brink of economic development.

General Santos medical facilities which signified their interest to take part in the study were the following: Mindanao Medical Center (MMC), General Santos City District Hospital (GSCDH), St. Elizabeth Hospital (SEH), General Santos Doctor's Hospital (GSDH), SOCKSARGEN County Hospital (SCH), and Puericulture Family Planning and Maternity Clinic (PFPMC). Pertinent data were also collected from the local National Statistics Office. Since there was no local Ethics Review Board as of the time the study was conducted, permission was secured from Hon. Rosalita Nunez, the Chairman of the Committee on Health of the Sanguniang Panglungsod (City Legislative Council) and Dr. Jacinto Maquilang, the City Health Officer. Letters of prior informed consent were also sent to the different hospital directors before data collection was done. Henceforth, retrospective study of in-patient records from the abovementioned hospitals was done for live births that occurred from January 2009 to December 2012. Live newborns diagnosed with at least $1 \mathrm{CA}$ were recorded and the prevalence of each anomaly was calculated. Obtained data from the selected hospitals were the number of CAs, CMDs, live births and the birth defect prevalence rates from 20092012.

\section{Results}

Shown in Table 1 is a listing of health-related statistics recorded in the city from 2009-2012. These data were taken from 6 private hospitals, 4 maternal clinics and 1 government-managed hospital in the city. For this study, 6 medical institutions viz. GSCDH, GSDH, MMC, SEH, SCH and PFPMC furnished the researchers with needed data. GSDH, SEH, MMC, and SCH are tertiary private hospitals while GSCDH is a government-managed hospital catering to indigent patients. This latter public hospital has the largest number of births per year among the medical facilities (Table 2). On the other hand, PFPMC is a government-managed maternity clinic which has the second largest number of births per year except for 2010.

Table 1. Related Health Statistics of General Santos City

\begin{tabular}{lcccc}
\hline & 2009 & 2010 & 2011 & 2012 \\
\hline Total Population of & 567,588 & 587,623 & 608,634 & 567,645 \\
General Santos City & & & & \\
Number of Births & 14,566 & 14,603 & 15,911 & 15,905 \\
Number of Deaths & 2,004 & 2,666 & 2,544 & 2,842 \\
Infant Mortality Rate & 6.17 & 8.76 & 8.42 & 11.56 \\
(IMR/1000LB) & & & & \\
Number of Infant Deaths & 90 & 128 & 134 & 184 \\
Number of Fetal Deaths & 56 & 54 & 77 & 49 \\
Facility Based Deliveries & $48.00 \%$ & $43.00 \%$ & $58.00 \%$ & $61.76 \%$ \\
\hline Source: National Statistics Office, General Santos City & &
\end{tabular}

Table 2. Total Number of Births in Selected Medical Facilities in General Santos City

\begin{tabular}{lcccc}
\hline & $\mathbf{2 0 0 9}$ & $\mathbf{2 0 1 0}$ & $\mathbf{2 0 1 1}$ & $\mathbf{2 0 1 2}$ \\
\hline $\begin{array}{l}\text { General Santos City District } \\
\text { Hospital (GSCDH) }\end{array}$ & 3,424 & 3,844 & 4,449 & 4,244 \\
$\begin{array}{l}\text { Saint Elizabeth Hospital (SHE) } \\
\text { General Santos Doctors' }\end{array}$ & 528 & 612 & 609 & 686 \\
$\begin{array}{l}\text { Hospital(GSDH) } \\
\text { Mindanao Medical }\end{array}$ & 542 & 512 & 546 & 544 \\
$\begin{array}{l}\text { Center(MMC) } \\
\text { SOCSKSARGEN County }\end{array}$ & 2457 & 284 & 241 & 255 \\
$\begin{array}{l}\text { Hospital(SCH) } \\
\text { JCC. Sr. Puericulture Center }\end{array}$ & 566 & 517 & 680 & 9796 \\
$\begin{array}{l}\text { Maternity Clinic (PFPMC) } \\
\text { General Santos City (Total) }\end{array}$ & 14,566 & 14,603 & 15,911 & 15,905 \\
\hline
\end{tabular}

\section{Prevalence of Congenital Anomalies from 2009 - 2012}

A total of 109 CA cases were reported in the selected General Santos City medical facilities during the years 20092012. A breakdown of the 109 reported cases in this study revealed $53.21 \%$ isolated CAs, $21.10 \%$ combination of CAs (more than 1 malformation in single organ category in an individual), $17.43 \%$ multiple CAs (more than 1 malformation in different organ systems in one individual) while the remaining $8.26 \%$ constituted syndromic CAs (combinations of developmental defects sharing a common etiology). Of the 109 cases, 71 were recorded from GSCDH and 21 cases were from GSDH (Figure 1).

Table 3 reveals the nature of CAs observed in Gen. Santos City during the inclusive years of the study. A cumulative number of 30 cases were observed for limb deformities which translates roughly to 11.63 patients/10,000 
births. These deformities included clubfoot (8 cases), polydactyly (6 cases), limb deficiencies (5 cases), syndactyly (1 case), brachysyndactyly (1 case), amniotic band syndrome ( 2 cases), underdeveloped limbs (2 cases), calcaneovalgus feet - unspecified ( 1 case), and other limb deformities (2 cases).

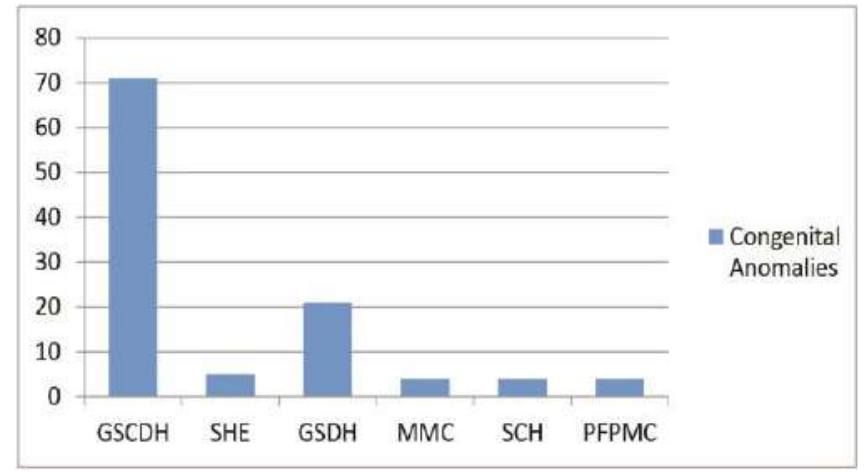

Figure 1. Reported Congenital Anomalies in major Medical Facilities in General Santos City from 2009- 2012.

Table 3. Top 10 Birth Defects Prevalence Rates from 20092012.

\begin{tabular}{ccc}
\hline $\begin{array}{c}\text { Type } \\
\text { of CAs }\end{array}$ & $\begin{array}{c}\text { Number } \\
\text { of Cases }\end{array}$ & $\begin{array}{c}\text { Rate per } \\
\mathbf{1 0}, \mathbf{0 0 0} \text { births }\end{array}$ \\
\hline Limb Deformities & 30 & 11.63 \\
Orofacial Clefts & 25 & 9.69 \\
NTD ( Neural Tube Defects) & 13 & 5.04 \\
Atresia & 9 & 3.49 \\
Down Syndrome & 8 & 3.10 \\
Hydrocephalus & 7 & 2.71 \\
Gastroschisis & 3 & 1.16 \\
\hline
\end{tabular}

Oro-facial clefts followed closely at 25 cases and a prevalence rate of $9.69 / 10,000$ births. The variable nature of oro-facial clefting was demonstrated in the following percentages obtained: cleft lip made up $24 \%$ of total orofacial cleft cases while cleft lip/palate and cleft palate comprised $60 \%$ and $16 \%$ of reported cases, respectively. Furthermore, $80 \%$ of orofacial clefts were observed to be isolated cases whereas the remaining $20 \%$ in the study were either in combination with other malformations found in a single organ category or in multiple congenital anomalies.

There were 13 identified neural tube defect (NTD) cases in this study of which $53.84 \%$ were meningocele and $46.15 \%$ were anencephaly cases. Other CA cases observed were 9 cases of atresia, of which $77 \%$ of the neonates had imperforate anus while the remaining malformations were due to duodenal atresia and an uncategorized atresia. The study also identified 8 cases of Down syndrome with a prevalence rate of $3.10 / 10,000$ live births. This prevalence rate is considerably lower when compared to an earlier study conducted by Padilla et al in Manila and Zapico et al in General Santos City.,3 Furthermore, the prevalence rate of congenital hydrocephalus in the study was 2.71/10, 000 live births which was slightly higher than the prevalence rate reported by the Philippine Birth Defects Registry Project. ${ }^{5}$

\section{Congenital Metabolic Disorders (CMDs) Detected during Neonate Screening}

Figure 2 shows the number of neonates manifesting any form of CMD during the study period. As can be seen from the graph, there was a drastic increase in CMD cases from 2010 to 2012 for GSCDH. PFPMC, as a lying in clinic, was not included in the table since it did not have data on these specific metabolic disorders.

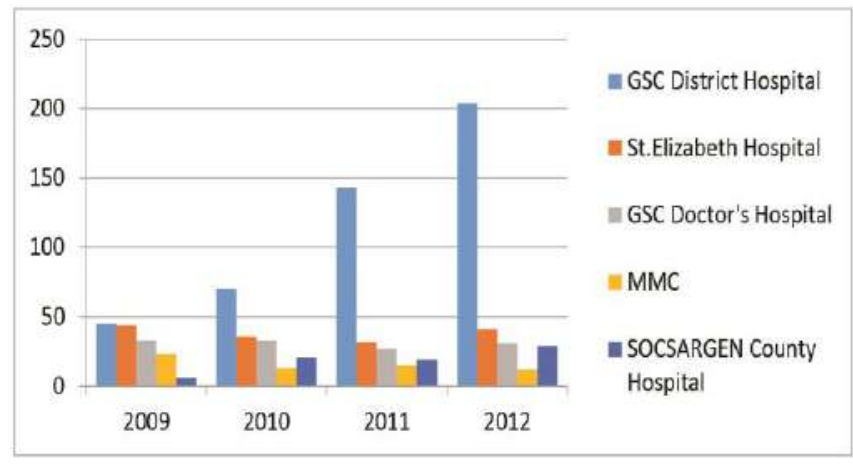

Figure 2. Congenital Metabolic Disorders Among Neonates in General Santos City

Shown in Table 4 are the numbers of live newborns exhibiting CMDs. A total of 829 cases of G6PD deficiency were reported in the selected medical facilities from 20092012. Starting from 2010 up to 2012, there was a remarkable increase in the number of G6PD deficiency cases in GSCDH when compared with the other medical facilities. G6PD deficiency, the most common metabolic condition observed in this study, is an X-linked recessive disorder characterized by enzyme defects of red blood cells. ${ }^{6}$ Usual symptoms of G6PD deficiency include hemolytic anemia, tea-colored urine, jaundice and pallor. ${ }^{7}$ G6PD patients also have decreased RBC lifespan and may have cataract formation.

Table 4 also shows a cumulative number of 16 cases of neonates born with $\mathrm{CH}$. Of this number, 6 cases were reported in SCH in 2012 while the remaining cases were noted in MMC and GSCDH. $\mathrm{CH}$, which is due to a deficiency of thyroid hormones present at birth, is usually caused by thyroid dysgenesis or defects in thyroid metabolism. Physiological consequences if left untreated are severe mental retardation, neurologic complications, and physical delays. 
Table 4. Congenital Metabolic Disorders Detected among Live-born Neonates in Gen. Santos City (2009-2012)

\begin{tabular}{|c|c|c|c|c|c|c|c|c|c|c|c|c|c|c|c|c|c|c|c|c|}
\hline \multirow{2}{*}{$\begin{array}{l}\text { Medical } \\
\text { Facilities }\end{array}$} & \multicolumn{4}{|c|}{ G6PD Def } & \multicolumn{4}{|c|}{$\begin{array}{c}\text { Congenital } \\
\text { Hypothyroidism }\end{array}$} & \multicolumn{4}{|c|}{$\begin{array}{l}\text { Congenital Adrenal } \\
\text { Hyperplasia }\end{array}$} & \multicolumn{4}{|c|}{ Galactosemia } & \multicolumn{4}{|c|}{ PKU/HPA } \\
\hline & ڤ્ণે & 유ํ & تี่ & สี้ & ठे̀े & 오ํ & تี่ & ָับ & ڤ્̀े & ㅇํํ & تี่ & ฮี & ठิे & ㅇํํ & $\overrightarrow{\text { ते }}$ & ัำ & ठ્ेે & ㅇํํ & تี่ & ปี \\
\hline GSCDH & 41 & 65 & 134 & 201 & 1 & 1 & 4 & - & 3 & 5 & 3 & - & - & 1 & - & 1 & - & 2 & 2 & 3 \\
\hline SEH & 44 & 36 & 32 & 41 & - & - & - & - & - & - & - & - & - & - & - & - & - & - & - & - \\
\hline GSDH & 31 & 31 & 27 & 30 & - & - & - & - & 2 & 2 & - & 1 & - & - & - & - & - & - & - & - \\
\hline MMC & 20 & 10 & 13 & 11 & 1 & 1 & 2 & - & 2 & 1 & - & - & - & - & - & 1 & - & - & - & - \\
\hline $\mathrm{SCH}$ & 6 & 17 & 18 & 21 & - & - & 1 & 6 & 2 & 2 & - & 1 & - & - & - & & - & - & - & - \\
\hline
\end{tabular}

From 2009 to 2011, GSCDH had an upsurge in the number of CAH cases though there was no report in 2012 for this metabolic disorder. Other $\mathrm{CAH}$ cases were also observed in $\mathrm{SCH}, \mathrm{GSDH}$ and $\mathrm{MMC}$. $\mathrm{CAH}$ is a genetic condition that inhibits hormone secretion by the adrenal glands. All forms of $\mathrm{CAH}$ follow autosomal recessive patterns of inheritance and $90 \%$ of reported cases is caused by 21-dyhroxylase deficiency (21-DD) which in turn causes hyperplasia of the adrenal cortex. Another consequence of $\mathrm{CAH}$ is excessive production of androgens which causes premature puberty in males and virilization in females. Furthermore, only 3 cases of galactosemia were recorded in MMC and GSCDH from 2009 to 2012. GAL is an autosomal recessive disorder in carbohydrate metabolism which results in elevated galactose levels due to deficiencies for galactosemetabolizing enzymes. ${ }^{7}$ Signs and symptoms in untreated patients include failure to thrive, feeding problems, hepatocellular damage, bleeding, sepsis and cataracts. Finally, 7 cases of PKU and HPA were noted in GSCDH during the years 2010-2012. HPA and PKU are autosomal recessive disorders characterized by high levels of phenylalanine. The disorder is caused by phenylalanine hydroxylase $(\mathrm{PAH})$ or tetrahydrobiopterin (BH4) deficiency which deficiency causes accumulation of phenylalanine in the blood and other tissues and results in the disruption of transport of certain amino acids across the blood-brain barrier. Clinical symptoms include distinct musty smell, developmental delay, mental retardation, microcephaly, seizure, eczema, and behavioral abnormalities. ${ }^{7}$

\section{Discussion}

This study was undertaken to improve the current state of knowledge available on birth prevalence of CA/CMD. While major hospitals in the city had started newborn screening in the early 2000s, there was no effort done to consolidate gathered data to get a true picture of the local prevalence of CAs and CMDs. Therefore, publications like this will evoke a sense of awareness and compassion about this long marginalized group of patients. This will also guide pediatricians and other primary care practitioners as to the special needs of these infants and the management options suitable for them.
In terms of CAs, a positive finding was the reduced number of infants born with anencephaly. This NTD has been known to contribute significantly to neonate mortality. ${ }^{6}$ This lowered number of anencephaly in this report when compared to that obtained by Zapico and her colleagues in General Santos City can be ascribed to increased folic intake among pregnant women which is a positive outcome of the government's massive information drive about the importance of nutrient balance during this stage of conception. $3,8,9$

Another noteworthy observation was the preponderance of CAs and CMDs in GSCDH, a governmentmanaged hospital that collects minimal fees for medical procedures and medicines. This hospital also recorded highest numbers for G6PD def/ $\mathrm{CAH}$ and was the only medical facility with PKU/HPA cases during these inclusive years. While these numbers may suggest a tentative association between CA/CMD incidences and poverty, the collected data are far too limited to lend credence to such speculations. Moreover, sweeping conclusions about the purported effects of economic deprivations are unwarranted especially with the absence of data about maternal malnutrition and exposure to harmful agents during pregnancy. On the other hand, Grewal et al, Pawluk et al and Clark et al, while studying the effects of nutritional deficiencies on various CAs, acknowledged that the harmful effects of economic deprivations (especially nutritional) and unknowing exposure (possibly to teratogens) could not be totally discounted. ${ }^{10,11,12}$

These perplexing claims provide ample justification for more CA/CMD prevalence studies in GSC especially since home deliveries constitute almost $40 \%$ of total live births in the city. These types of studies will be valuable to government agencies, the medical community and other sectors of society concerned with health and human resources. Information from the study can be used to draft certain policies or to source out funds from funding agencies so that the plight of these unfortunate individuals can be improved. 


\section{Conclusion}

While the study only covered selected hospitals in General Santos City, it may serve as a microcosm of $\mathrm{CA} / \mathrm{CMD}$ cases of the country at large. Major challenges in newborn screening initiatives in the Philippines are extreme poverty of majority of the Filipinos and their lack of comprehensive health care insurance. Moreover, since only facility-based deliveries were included in the study, these results do not give an accurate account of CA/CMD incidence in the city. Another factor to reckon with is the general ignorance of pregnant women about the harmful effects of teratogenic drugs, substances and other exposures on their developing babies. It is imperative therefore that local birth registries especially at the barangay level be established and that education of women about the perils of indiscriminate drug use and exposure to potentially teratogenic agents be undertaken so that they could take necessary precaution especially during the early stages of pregnancy.

\section{Acknowledgment}

The authors convey their most profound thanks to the participating hospitals for allowing the researchers access to hospital records. Hon. Rosalita T. Nunez, PhD of the Sangguniang Panglungsod, General Santos City Health Officer and Dr. Jacinto Maquilang of the General Santos City District Hospital (GSCDH) are also acknowledged for supporting this study.

\section{References}

1. World Health Organization. Congenital anomalies [Online]. 2012 [cited 2013 June]. Available from http://www.who.int/mediacentre/ factsheets/fs370/en/.

2. Newborn Health in the Philippines: A Situation Analysis. Published by the Basics Support for Institutionalizing Child Survival Project (BASICS II) for the United States Agency for International Development. Arlington, Virginia, June 2004.

3. Zapico FL, Aguilar CH, Lasalita AT, Jamerlan R. Congenital anomalies among live-born neonates in five major hospitals (2006-2008), Genera Santos City, Philippines. University of Southern Mindanao Research and Development Journal. 2010: 18(1):27-31.

4. Philippine Statistics Authority 2010 [Online]. 2013 [cited 2013 June] Available from https://psa.gov.ph/sites/default/files/attachments/hsd/ pressrelease/SOCCSKSARGEN.

5. Padilla CD, Cutiongco EM, Sia JM. Birth defects ascertainment in the Philippines. Southeast Asian J Trop Med Public Health. 2003; 34 Suppl 3:239-43.

6. David-Padilla C, Cutiongco-de la Paz EM, Cavan BC, et al. Establishment of the Philippine birth defects surveillance. Acta Med Philipp. 2011; 25(4):12-19.

7. Chiong MA, Fagela-Domingo C, Capistrano-Estrada S, Gepte MB. Basic information for physicians. Published by the Newborn Screening Reference Center National Institutes of Health. October 2010.

8. Department of Health. Women's health and safe motherhood project [Online]. 2010 [cited 2013 June]. Available from www.doh.gov.ph/node/1076.html.

9. Vera M. DOH maternal health program. For all your nursing needs [Online]. 2012 [cited 2013 June]. Available from www.nurseslabs.com/doh-maternal-health-program.

10. Grewal J, Carmichael SL, Song J, Shaw GM. Neural tube defects: an analysis of neighbourhood- and individual-level socio-economic characteristics. Paediatr Perinat Epidemiol. 2009; 23(2):116-24.

11. Clark JD, Mossey PA, Sharp L, Little J. Sociooeconomic status and orofacial clefts in Scotland, 1989-1998. Cleft Palate Craniofac J. 2003;40(5):481-5

12. Pawluk MS, Campaña H, Gili JA, et al. Adverse social determinants and risk for congenital anomalies. Arch Argent Pediatr. 2014; 112(3):215-23.

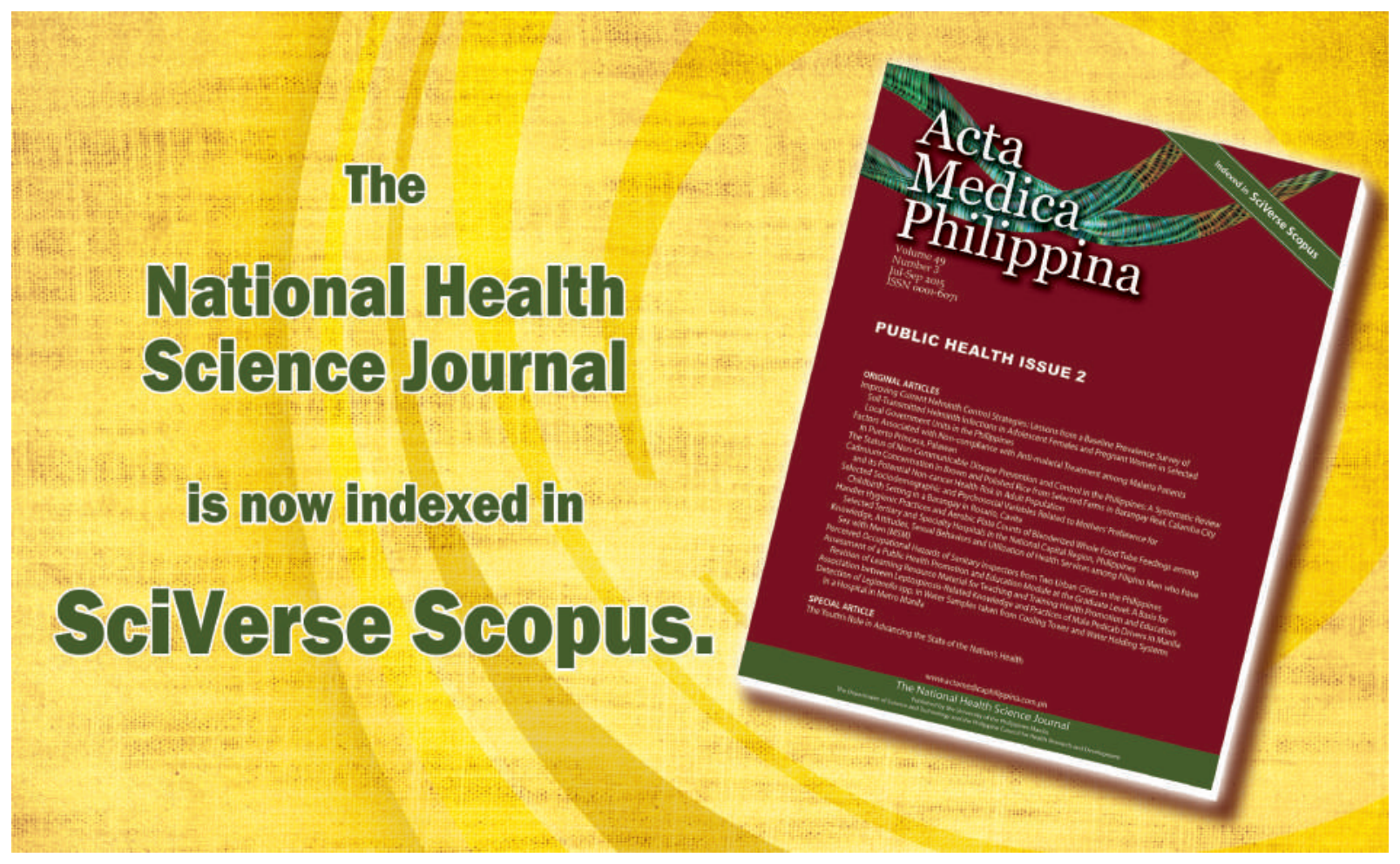

